\title{
Memory and Spin Injection Devices Involving Half Metals
}

\author{
M. Shaughnessy, Ryan Snow, L. Damewood, and C. Y. Fong \\ Department of Physics, University of California, Davis, CA 95616-8677, USA \\ Correspondence should be addressed to M. Shaughnessy, mickeyshaughnessy@gmail.com
}

Received 27 October 2010; Accepted 22 January 2011

Academic Editor: Zhi Li Xiao

Copyright ( $) 2011$ M. Shaughnessy et al. This is an open access article distributed under the Creative Commons Attribution License, which permits unrestricted use, distribution, and reproduction in any medium, provided the original work is properly cited.

\begin{abstract}
We suggest memory and spin injection devices fabricated with half-metallic materials and based on the anomalous Hall effect. Schematic diagrams of the memory chips, in thin film and bulk crystal form, are presented. Spin injection devices made in thin film form are also suggested. These devices do not need any external magnetic field but make use of their own magnetization. Only a gate voltage is needed. The carriers are $100 \%$ spin polarized. Memory devices may potentially be smaller, faster, and less volatile than existing ones, and the injection devices may be much smaller and more efficient than existing spin injection devices.
\end{abstract}

\section{Introduction}

The discovery of giant magnetoresistance in 1988 [1-3] led to a new era of using electron spin or both spin and charge as operating paradigm for memory and sensor devices [4]. For example, IBM developed MRAM by using metallic layered structures [5]. In 2000, the concept of spintronics was conceived [6] to merge the spin operations with semiconductors. Since then, the focus has been on three primary new challenges: (1) the search for new spintronic materials, (2) the fabrication of new spintronic devices, operable at room temperature, for logic, communication, and storage as well as devices that integrate these functions, such as spin transistors, and (3) the design of qubits for quantum computing.

There has been significant progress in finding new spintronic materials. Notably, magnetic semiconductors in which transition metal elements alloy with III-V [7], IIVI [8] semiconductors, Heusler alloys [9], compounds of transition metalpnictides [10,11], and oxides [12-15] have been reported or predicted. Recently, groups in France and Poland grew single magnetically doped quantum dots and probed the spin orientation of the transition metal element, Mn, using optical methods $[8,16]$, in anticipation that these magnetic quantum dots can lead to quantum computing components. It remains a challenging task to fabricate spintronic devices operable at room temperature with effective spin injection $[17,18]$.
In this paper, we propose a new memory device as well as a spin injection device made of half metals based on the anomalous Hall effect (AHE) [19]. These devices can help address some of the basic challenges for spintronics, namely, spin-polarized communication and memory storage, by showing how half-metallic materials can be useful. In Section 2, we will discuss the materials aspects relevant to the suggested devices. In Section 3, we briefly review the Hall effect including the AHE. Our proposed devices and their basic principles will be described in Section 4. Finally, a summary will be presented in Section 5 .

\section{Material Aspect}

We propose devices made of half metals and use CrAs as an example for demonstration. Any half-metallic material should show similar properties and should have qualitatively similar performance in our proposed devices.

CrAs can assume the metastable zinc blende, ZB, crystal structure, which may be viewed as a face-centered-cubic structure. It has been grown in thin film form on substrates with appropriate lattice constant matching, such as GaAs [10]. ZB CrAs is predicted to be a half metal $[10,11]$ using both the linear combination of augmented plane wave [10] and the VASP $a b$ initio electronic structure code [12-15]. Both calculations give a magnetic moment of 
$3.0 \mu_{\mathrm{B}} /$ formula-unit, where $\mu_{\mathrm{B}}$ is the Bohr magneton, which corresponds to a saturation magnetization of $550 \mathrm{kA} / \mathrm{m}$. Further details can be found in Pask et al. [11]. The measured coercivity is less than 100 Oe; a Curie temperature was estimated to be higher than $400 \mathrm{~K}$ [10]. Other calculations [16] suggest that $\mathrm{ZB}$ CrAs maintains its half-metallic character even near the interface with GaAs. Figure 1 shows the unit cell of CrAs in the ZB structure.

Theoretically, a half metal should satisfy two conditions [17]: first, the magnetic moment per unit cell is an integer, and, secondly, the density of states (DOS) for one spin channel should show metallic properties while the other channel exhibits a gap between the top of the valence bands and the bottom of the conduction bands-a semiconducting character. The Fermi energy $E_{F}$, of the metallic channel falls in the gap of the semiconducting channel; therefore, the spin polarization at $E_{F}$ is $100 \%$. The reason for the integer moment is because the total number of electrons is an integer and also the number of electrons occupying the valence bands is an integer, and so the number of electrons in the metallic channel should be an integer.

The bulk band structure and the calculated DOS of the two spin channels for CrAs are shown in Figures 2 and 3 , respectively. The band structure shows that the Fermi energy, $E_{F}$, set to be zero, intersects the antibonding states of the d-p hybridized bands at the $\Gamma(\mathbf{k}=0)$ point, to where the state drops down from the unoccupied states. The detailed physical processes for forming a half metal in the ZB structure has been given by Fong et al. [18]. The majority spin channel possesses both a localized moment and magnetized itinerate electrons. The localized moments are manifested in the DOS of the majority spin channel (Figure 3(a)) by the peaks exhibited between $-125 \mathrm{mHa}$ to $E_{F}$. The semiconducting feature in the down spin channel is clearly exhibited in Figure 3(b).

\section{The Hall Effect}

The proposed devices are based on the AHE. The "anomalous" part characterizes the additional magnetic induction, equation (1), when a sample is ferromagnetic. The physical origin is still controversial [19]. Hirsch [20] pointed out that the magnetization of a ferromagnetic sample could cause an extra Hall field. The useful review by Nagaosa [21] covers theoretical and experimental aspects of the AHE and includes an especially relevant discussion of the AHE in Heusler alloys, some of which are also half metallic.

Based on classical electromagnetic theory, the $\mathbf{B}$-field is given by

$$
\mathbf{B}=\mathbf{H}+4 \pi \mathbf{M},
$$

where $\mathbf{H}$ is an applied magnetic field, and $\mathbf{M}$ is the magnetization. A nonzero $\mathbf{B}$ can be produced even when $\mathbf{H}$ is zero. Thus, there will be a Hall effect due to $\mathbf{M}$ if an external electric field is applied perpendicular to $\mathbf{M}$, that is, no external magnetic field is needed.

For CrAs, the DOS (Figures 3(a) and 3(b)) shows most of the d-states in both majority and minority spin states

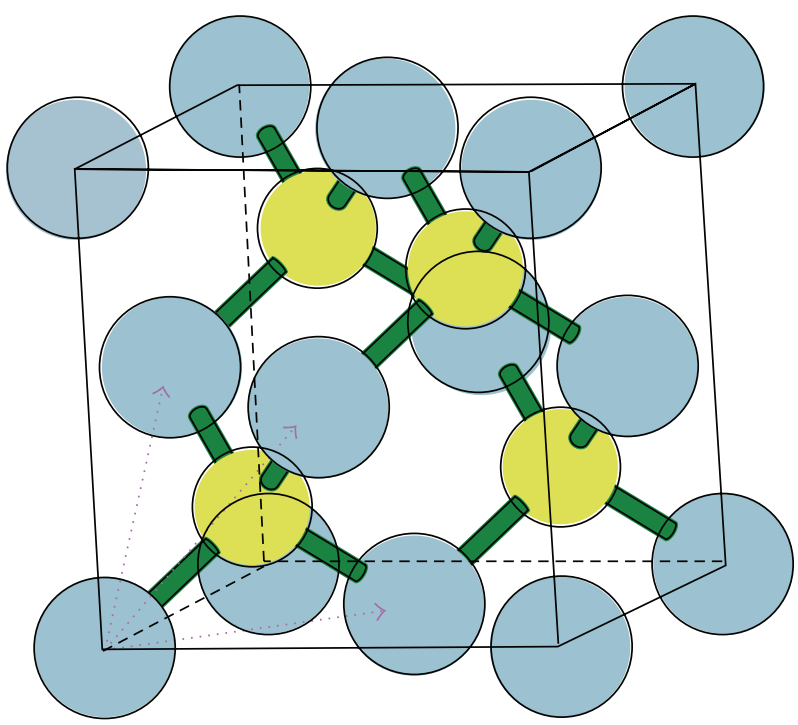

FIGURE 1: CrAs in a conventional cubic cell. Cr is shown in cyan and As is yellow.

located $50 \mathrm{mHa}$ below $E_{F}$. The exchange splitting between the two spin channels and the associated difference in occupation causes the localized magnetization of the sample. The electrons at $E_{F}$ are the mobile carriers. Their response to an external applied electric field (along the $z$-direction) and $\mathbf{M}$ is the AHE. In a slab geometry with the magnetization in the plane (the $x$-direction) and an applied electric field out of the plane, there will be a voltage developed due to the motion of the conduction electrons. For a metal, a difference of charge accumulation develops between two edges of the slab (the $y$-direction). In a half metal, both charge and spin show such differences, since the conduction electrons are completely polarized.

\section{The Proposed Devices}

4.1. Memory Device. The operation of the memory device can be illustrated as a thin film or in a layered structure. For the layered structure, we will use the cubic crystal as an example.

4.1.1. Thin Film Form. We use half-metallic CrAs as an example material. Other possible half metals include $\mathrm{CrO}_{2}$, $\mathrm{ZB} \mathrm{MnC}$, or MnSb. These should all be grown on appropriate substrates to ensure the thin films have the lattice constants and the $\mathrm{ZB}$ structure required to exhibit half-metallic properties. If we define the direction of the magnetization of the half-metallic ferromagnet (CrAs), $\mathbf{M}$, to be in the plane of the film, say the $x$-direction, it is possible to get an anomalous Hall voltage if an electric field, $\mathbf{E}$, is applied perpendicular to the film, in the $z$-direction. This geometry is shown in Figure 4.

The force due to the AHE drives the conduction electrons in the $y$-direction. Electrons inside the sample are accumulated at the front edge of the lower figure. Since these conduction electrons are completely spin-polarized, they will 


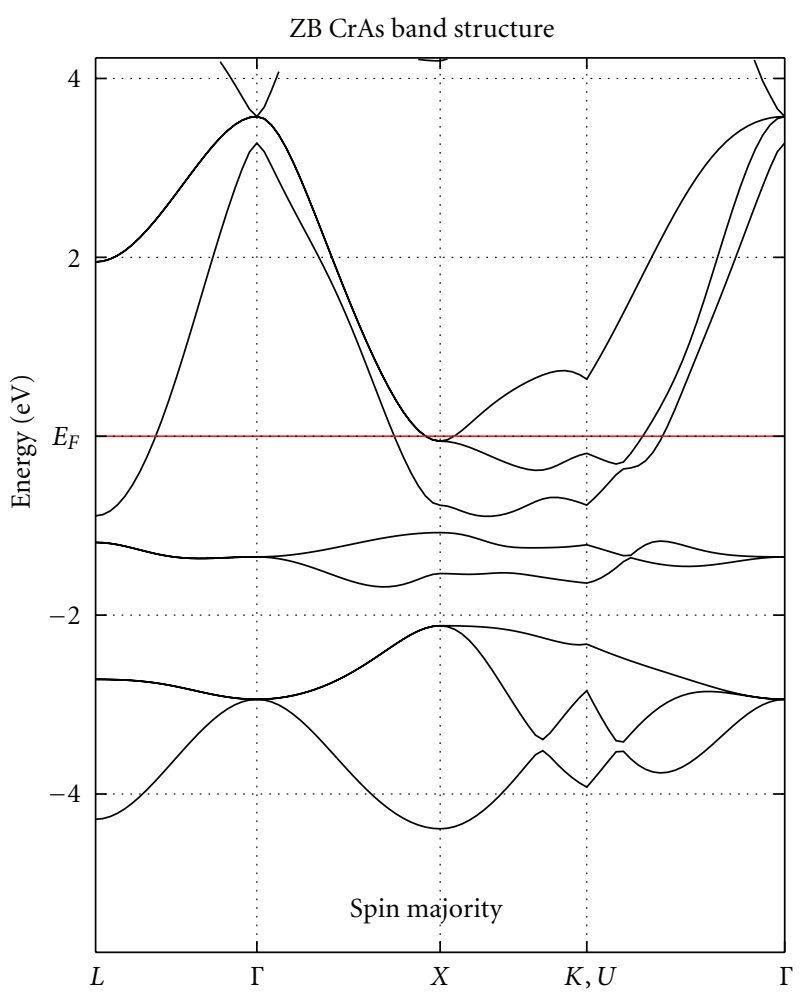

(a)

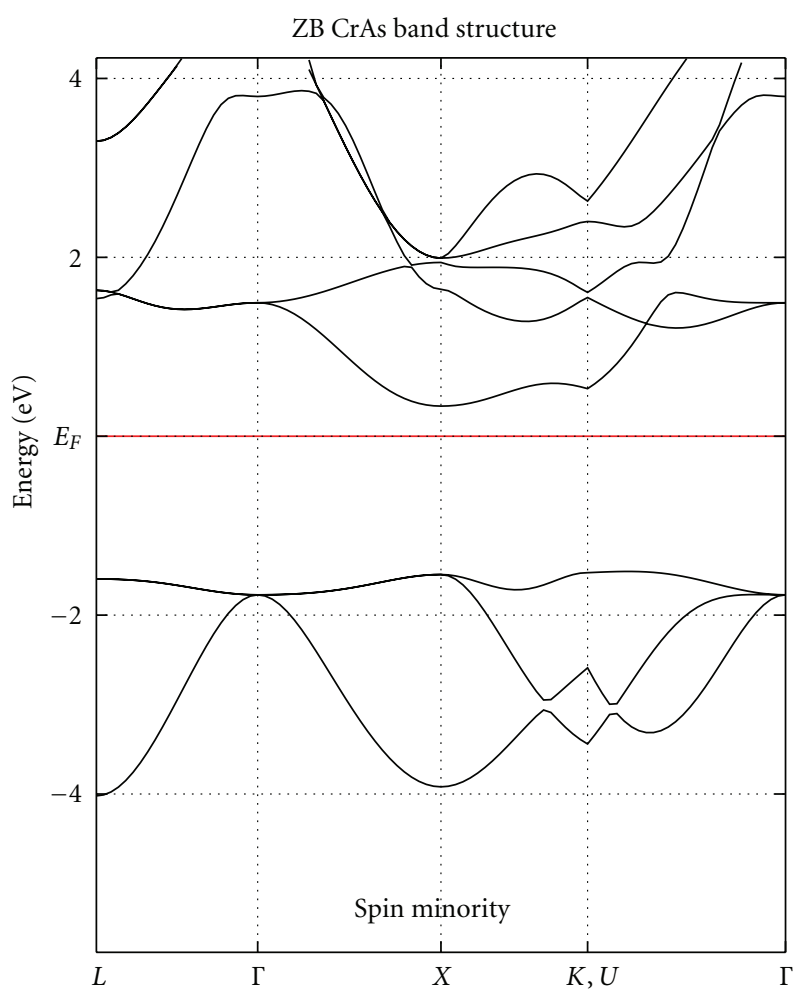

(b)

Figure 2: The bulk band structure of CrAs.

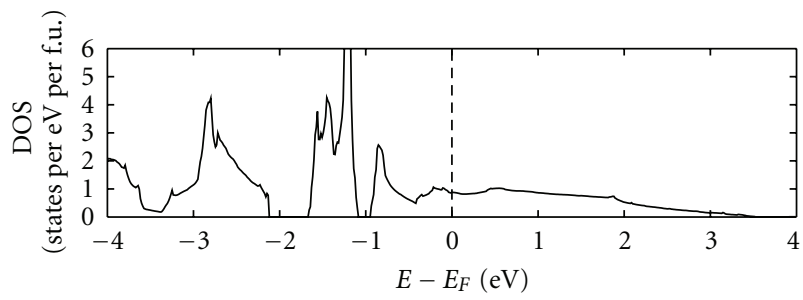

(a)

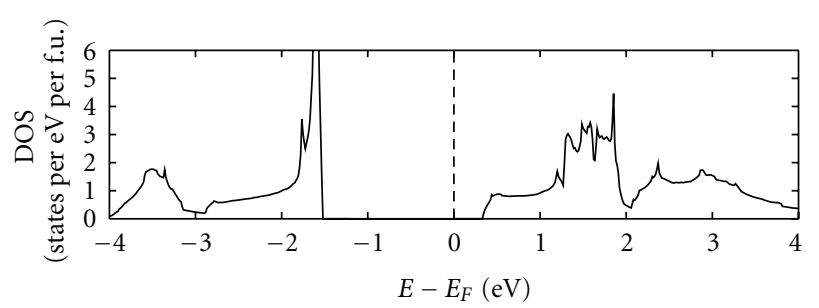

(b)

Figure 3: CrAs density of states per hartree per cell. (a) The up spin channel, (b) the down spin channel.

produce an additional magnetic moment, $\Delta \mathbf{M}$, near the front edge. If a gate voltage controls $\mathbf{E}$ and the induced $\Delta \mathbf{M}$ changes the magnetic polarization in an adjacent material, then we have a device for memory. The adjacent material can be dilutely Mn doped GaAs, single Mn doped quantum dots, or in fact any soft ferromagnetic material. It is also possible to grow stripes instead of one large film form perpendicular to the $z$-direction. Then, one can place the quantum dots at the centers of stripes to enhance the effect of $\Delta \mathbf{M}$.

4.1.2. Layered Device. The memory effect can be more easily illustrated with a three-dimensional device in layered configuration as shown in Figure 5. The magnetization of the half metal is pointing in the $x$-direction, and the applied electric field is in the $z$-direction. $\Delta \mathbf{M}$ is the consequence of the anomalous Hall effect. The thin layer immediately below the half metal is a soft ferromagnet; below this is the pinning ferromagnet with its magnetization denoted by $\mathbf{M}_{p}$. The soft ferromagnet has its magnetization $\mathbf{M}^{\prime}$ parallel to $\mathbf{M}_{p}$ when $\mathbf{E}=0$. The pinning layer must have a large enough magnetization to initially align the $\mathbf{M}^{\prime}$ layer but not so large that the layer remains aligned when the gate voltage $V_{G}$ is turned on and the associated additional magnetization $\Delta \mathbf{M}$ is present. The depth of the insulating layer can be used to tune the strength of the interaction between the pinning and switching layer so that the above criteria is satisfied. The magnetization in the switching layer will reverse its direction to $-\mathbf{M}^{\prime}$ when there is a gate voltage $V_{G}$ and the associated $\Delta \mathbf{M}$. This setup combined with a spin-torquetransfer current may be necessary to switch the switching layer. If so, our configuration, which utilizes the AHE, may dramatically reduce the required current densities needed for devices using spin-torque. 


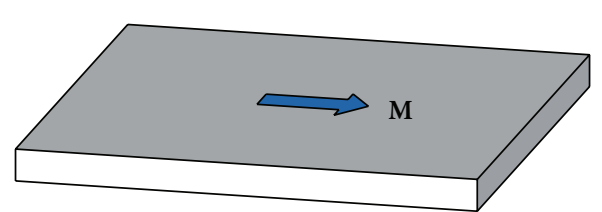

(a) No field

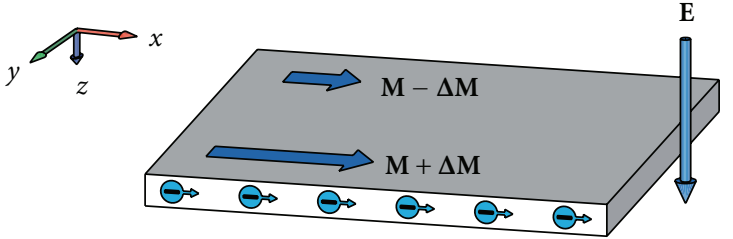

(b) With field

Figure 4: The thin film of CrAs (a) with the magnetic moment, $\mathbf{M}$, in the film. When an electric field is applied in the $z$-direction, the Hall electric field is in the $y$-direction. An accumulation of magnetic moment is shown by $\Delta \mathbf{M}(\mathrm{b})$.

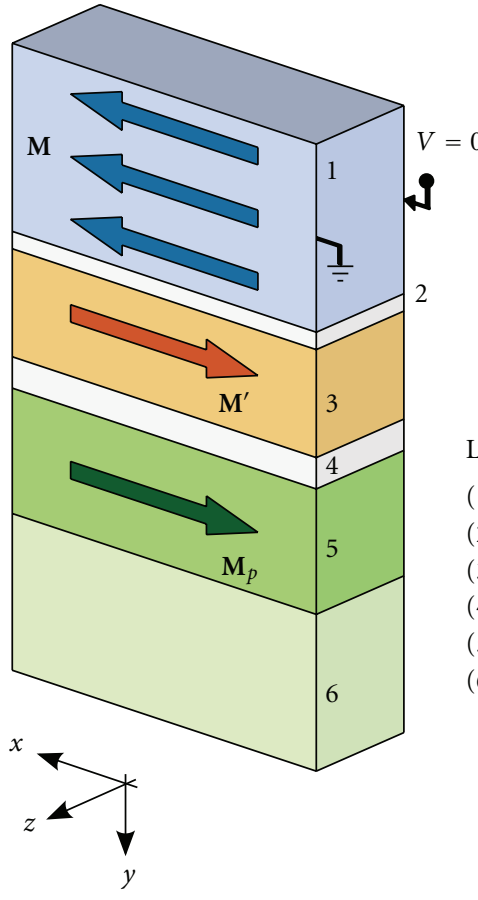

(a) Without gate voltage
Layers:

(1) HM layer

(2) Insulating spacer

(3) FM active layer

(4) Insulating spacer

(5) FM pinned layer

(6) AFM pinning layer

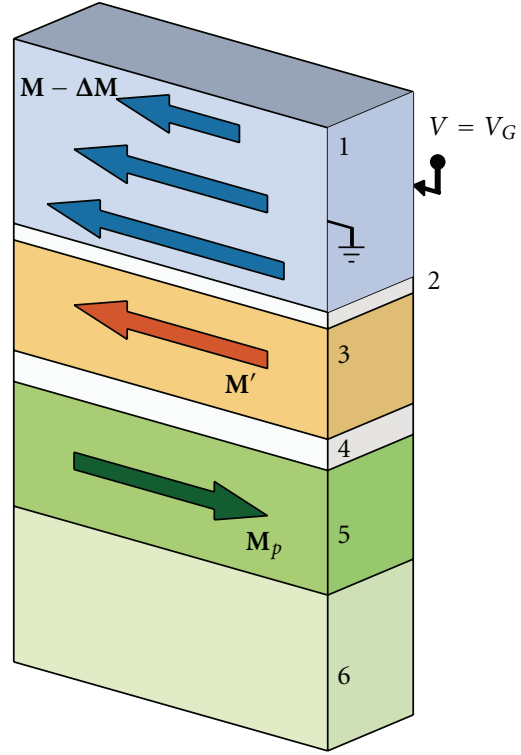

(b) With gate voltage

Figure 5: Configuration for a device made of a cubic half-metallic layer (HML) depicted in blue. The active ferromagnetic layer is shown in orange, the hard ferromagnetic pinned layer is shown in green, and the antiferromagnetic pinning layer is shown in light green. (a) Without a gate voltage, the magnetization of the active layer $\mathbf{M}^{\prime}$ aligns with the pinned layer magnetization $\mathbf{M}_{p}$ in $-x$-direction. (b) When the gate voltage is applied across the HM layer in the $z$-direction, the spin-polarized electrons are forced toward the bottom surface due to the anomalous Hall effect, thereby increasing the magnetization along the bottom surface of the HM layer by the amount $\Delta \mathbf{M}$. The increased magnetization in the $+x$-direction next to the top surface of the active layer causes the active layer magnetization to flip to the $+x$-direction.

4.2. Spin Injector. We can modify the shape of the film to have a device described above serving as a spin injector using the anomalous Hall effect. The modification is to have a sharp corner where the mobile carriers will accumulate. The configuration of the film and the external field are shown in Figure 6.

In Figure 6(a), similar to Figure 4, the magnetization, $\mathbf{M}$, is along the $x$-direction. An electric field, $\mathbf{E}$, due to a gate voltage is applied along the $z$-direction. Mobile electrons in the half metal accumulate in the $y$-direction. But the cornered shape of the sample forces the electrons out of the sample as in the tip of the scanning tunneling microscope. These electrons can be injected into doped GaAs, and will carry their spin moment in the direction of $\mathbf{M}$. It should be noted that for the spin-injector as well as the memory device described above the properties of the interface between the half metal and the GaAs or the switching layer, respectively, might effect the operation of both devices. Suitable combinations of half metals and active materials should be carefully tested in applications, although there are suggestions that $\mathrm{CrAs} / \mathrm{GaAs}$ interfaces will suffice [22].

Spin injection typically from a metallic source, such as the one proposed, into a semiconductor may suffer a problem due to spin flips from majority to minority spin in the accumulation region [23]. This has been alleviated using a thin spin-dependent interface resistance, typically a tunnel junction for the case of a metallic ferromagnet injector [19]. For the present case of a half-metallic source, however, the 


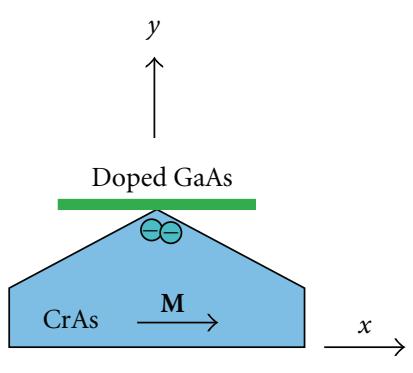

- $\mathrm{E}$

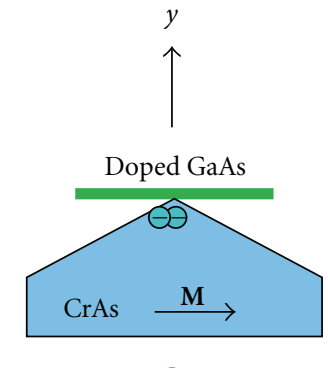

$\mathbf{E}$

(a)

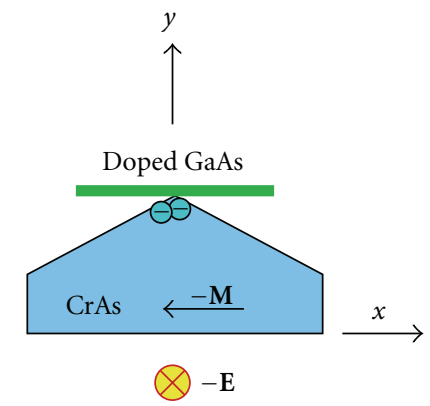

(b)

Figure 6: CrAs film is grown on top of a substrate (below the film). The $z$-axis is pointing out of the paper. The doped GaAs serves as the collector of the polarized current.

accumulation of spin at the tip of the injector will not lead to spin flips, since the minority spin DOS is gapped around the Fermi energy.

It is also possible to grow multiple sections of the films and orient $\mathbf{M}$ differently in each section as shown in Figure 6(b). By using a different gate voltage on each section, it is possible to inject spins with different polarizations. Because of the half-metallic property of the material in each section, spin-polarized electrons from a given section will only be injected for one sign of the gate voltage. Compare this with the same device made out of a metallic ferromagnet, such as $\mathrm{Fe}$, in which either sign of the gate voltage will cause both up and down spin polarized current to be injected. Therefore, the injection is less effective. The half-metallic property is crucial because it ensures that only one spin state is mobile and consequently only one polarization will originate from a given injector section. This may allow for much smaller and denser arrays of spin-injectors.

\section{Summary}

We propose two kinds of devices made of half metals. $\mathrm{ZB}$ CrAs is considered as an example because its $T_{C}$ is estimated experimentally above $400 \mathrm{~K}$ and its lattice constant is matched with GaAs. The basic physical mechanism for the devices is the anomalous Hall effect without the need of an external magnetic field. A memory device using the spin-polarized distribution of conduction electrons due to the Hall voltage as the switching mechanism is proposed. A spin injection geometry is suggested for thin-film spin injectors that serves to concentrate the spin current at a point is also suggested. The applied electric field to induce the Hall voltage can be controlled by a gate voltage. It is our hope that the proposed devices may stimulate someone to grow them.

\section{Acknowledgments}

This paper was supported in part by the National Science Foundation Grant no. ECCS-0725902 through TeraGrid resources provided by the TeraGrid Science Gateways program. This work was performed under the auspices of the
U.S. Department of Energy by Lawrence Livermore National Laboratory under Contract DE-AC52-07NA27344.

\section{References}

[1] M. N. Baibich, J. M. Broto, A. Fert et al., "Giant magnetoresistance of (001)Fe/(001)Cr magnetic superlattices," Physical Review Letters, vol. 61, no. 21, pp. 2472-2475, 1988.

[2] B. Dieny, V. S. Speriosu, S. S. P. Parkin, B. A. Gurney, D. R. Wilhoit, and D. Mauri, "Giant magnetoresistive in soft ferromagnetic multilayers," Physical Review B, vol. 43, no. 1, pp. 1297-1300, 1991.

[3] G. Binasch, P. Grünberg, F. Saurenbach, and W. Zinn, "Enhanced magnetoresistance in layered magnetic structures with antiferromagnetic interlayer exchange," Physical Review $B$, vol. 39, no. 7, pp. 4828-4830, 1989.

[4] G. A. Prinz, "Magnetoelectronics," Science, vol. 282, no. 5394, pp. 1660-1663, 1998.

[5] L. Savtchenko, B. N. Engel, N. D. Rizzo, M. F. Deherrera, and J. A. Janesky, "Method of writing to scalable magnetoresistance random access memory element," US patent, 6545906 B1, 2003.

[6] S. A. Wolf, D. D. Awschalom, R. A. Buhrman et al., "Spintronics: a spin-based electronics vision for the future," Science, vol. 294, no. 5546, pp. 1488-1495, 2001.

[7] H. Ohno and F. Matsukura, "Ferromagnetic III-V semiconductor: (Ga,Mn)As," Solid State Communications, vol. 117, no. 3, pp. 179-186, 2001.

[8] C. Le Gall, L. Besombes, H. Boukari, R. Kolodka, J. Cibert, and H. Mariette, "Optical spin orientation of a single manganese atom in a semiconductor quantum dot using quasiresonant photoexcitation," Physical Review Letters, vol. 102, no. 12, Article ID 127402, 2009.

[9] R. A. de Groot, F. M. Mueller, P. G. van Engen, and K. H. J. Buschow, "New class of materials: half-metallic ferromagnets," Physical Review Letters, vol. 50, no. 25, pp. 2024-2027, 1983.

[10] H. Akinaga, T. Manago, and M. Shirai, "Material design of half-metallic zinc-blende CrAs and the synthesis by molecular-beam epitaxy," Japanese Journal of Applied Physics, vol. 39, no. 11B, pp. L1118-L1120, 2000.

[11] J. E. Pask, L. H. Yang, C. Y. Fong, W. E. Pickett, and S. Dag, "Six low-strain zinc-blende half metals: an ab initio investigation," Physical Review B, vol. 67, no. 22, Article ID 224420, 7 pages, 2003. 
[12] VASP the GUIDE, Institut für Theoretische Physik of the Technishe Universtät, Wien, Austria, 1999.

[13] G. Kresse and J. Hafner, "Norm-conserving and ultrasoft pseudopotentials for first-row and transition elements," Journal of Physics: Condensed Matter, vol. 6, no. 40, pp. 8245-8257, 1994.

[14] G. Kresse and J. Furthmüller, "Efficient iterative schemes for ab initio total-energy calculations using a plane-wave basis set," Physical Review B, vol. 54, no. 16, pp. 11169-11186, 1996.

[15] Y. Ji, G. J. Strijkers, F. Y. Yang et al., "Determination of the spin polarization of half-metallic $\mathrm{CrO}_{2}$ by point contact Andreev reflection," Physical Review Letters, vol. 86, no. 24, pp. 5585$5588,2001$.

[16] M. Goryca, T. Kazimierczuk, M. Nawrocki et al., "Optical manipulation of a single $\mathrm{Mn}$ spin in a CdTe-based quantum dot," Physical Review Letters, vol. 103, no. 8, Article ID 087401, 2009.

[17] C. Chappert, A. Fert, and F. N. Van Dau, "The emergence of spin electronics in data storage," Nature Materials, vol. 6, no. 11, pp. 813-823, 2007.

[18] C. Y. Fong, M. C. Qian, K. Liu, L. H. Yang, and J. E. Pask, "Design of spintronic materials with simple structures," Journal of Nanoscience and Nanotechnology, vol. 8, no. 7, pp. 3652-3660, 2008.

[19] D. D. Awschalom and M. E. Flatté, "Challenges for semiconductor spintronics," Nature Physics, vol. 3, no. 3, pp. 153-159, 2007.

[20] J. E. Hirsch, "Overlooked contribution to the Hall effect in ferromagnetic metals," Physical Review B, vol. 60, no. 21, pp. 14787-14792, 1999.

[21] N. Nagaosa, J. Sinova, S. Onoda, A. H. MacDonald, and N. P. Ong, "Anomalous Hall effect," Reviews of Modern Physics, vol. 82, no. 2, pp. 1539-1592, 2010.

[22] K. Nagao, M. Shirai, and Y. Miura, "Ab initio calculations of zinc-blende Cras/GaAs superlattices," Journal of Applied Physics, vol. 95, no. 11, pp. 6518-6520, 2004.

[23] A. Fert, "Nobel lecture: origin, development, and future of spintronics," Reviews of Modern Physics, vol. 80, no. 4, pp. 1517-1530, 2008. 

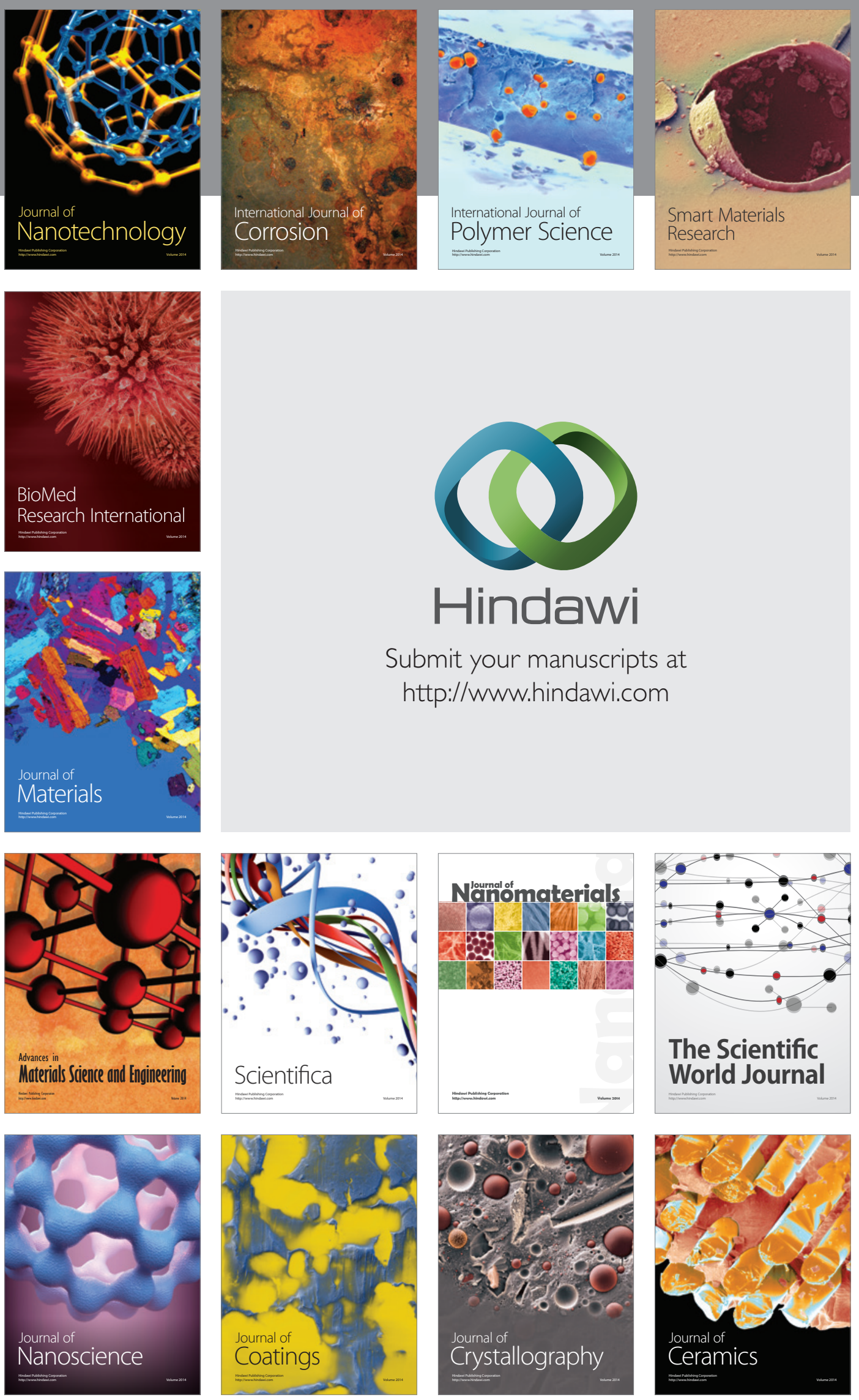

The Scientific World Journal

Submit your manuscripts at

http://www.hindawi.com

\section{World Journal}

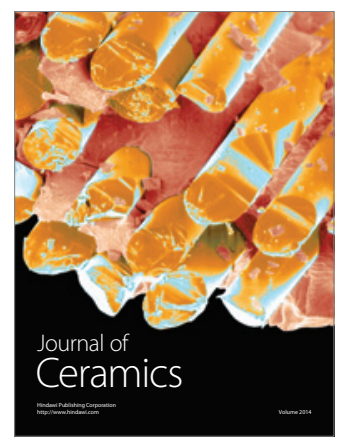

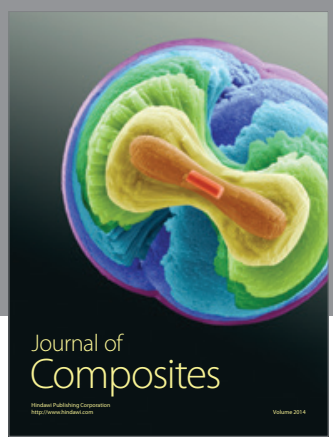
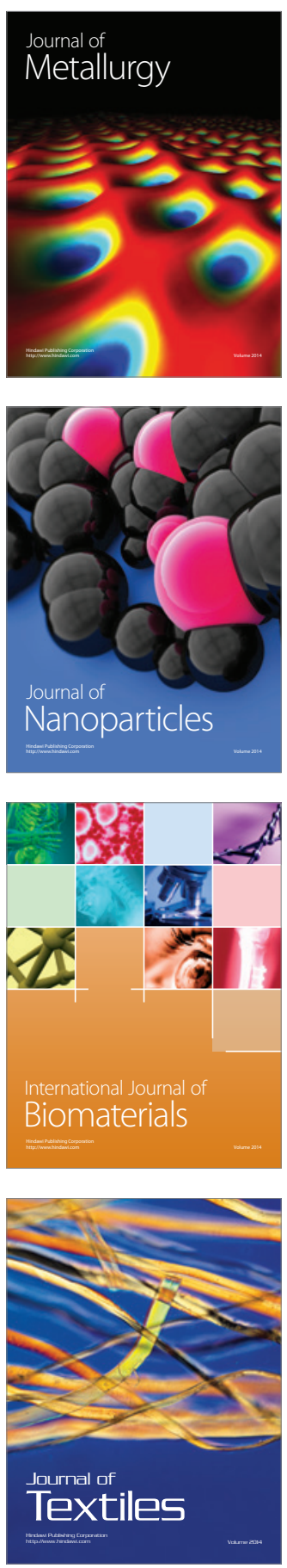